\title{
BMJ Open Risk factors and prognosis of seizures in adults with community-acquired bacterial meningitis in Denmark: observational cohort studies
}

Fredrikke Tove Birgitta Dam Larsen, ${ }^{1}$ Christian Thomas Brandt, ${ }^{2}$ Lykke larsen, ${ }^{3}$ Vibeke Klastrup, ${ }^{4}$ Lothar Wiese, ${ }^{5}$ Jannik Helweg-Larsen, ${ }^{6}$ Mette Riber, ${ }^{7}$ Birgitte Rønde Hansen, ${ }^{7}$ Christian Østergaard Andersen, ${ }^{8}$ Henrik Nielsen, ${ }^{1}$ Jacob Bodilsen, ${ }^{1}$ On behalf of the DASGIB study group
To cite: Larsen FTBD, Brandt CT, larsen $\mathrm{L}$, et al. Risk factors and prognosis of seizures in adults with communityacquired bacterial meningitis in Denmark: observational cohort studies. BMJ Open 2019;9:e030263. doi:10.1136/ bmjopen-2019-030263

- Prepublication history for this paper is available online. To view these files please visit the journal online (http://dx.doi org/10.1136/bmjopen-2019030263).

Received 7 March 2019 Revised 24 May 2019 Accepted 10 June 2019
Check for updates

(C) Author(s) (or their employer(s)) 2019. Re-use permitted under CC BY-NC. No commercial re-use. See rights and permissions. Published by BMJ.

For numbered affiliations see end of article.

Correspondence to Dr Jacob Bodilsen; Jacob.bodilsen@rn.dk

\section{ABSTRACT}

Objective To examine predefined risk factors and outcome of seizures in community-acquired bacterial meningitis (CABM).

Design Observational cohort studies

Setting Denmark

Participants In the derivation cohort, we retrospectively included all adults ( $>15$ years of age) with CABM in North Denmark Region from 1998 to 2014 and at Hvidovre and Hillerød hospitals from 2003 to 2014. In the validation cohort, we prospectively included all adults ( $>18$ years of age) with CABM treated at all departments of infectious diseases in Denmark from 2015 to 2017.

Primary and secondary outcome measures In the derivation cohort, we used modified Poisson regression to compute adjusted relative risks (RRs) with $95 \%$ confidence intervals for predefined risk factors for seizures during CABM as well as for risks of death and unfavourable outcome assessed by the Glasgow Outcome Scale score (1-4). Next, results were validated in the validation cohort. Results In the derivation cohort $(n=358)$, risk factors for seizures at any time were pneumococcal aetiology (RR 1.69, 1.01-2.83) and abnormal cranial imaging (RR $2.27,1.46-3.53)$, while the impact of age $>65$ years and immunocompromise was more uncertain. Examining seizures occurring after admission, risk factors were abnormal cranial imaging (RR 2.23, 1.40-3.54) and immunocompromise (RR 1.59,1.01-2.50). Seizures at any time were associated with increased risks of in-hospital mortality (RR 1.45, 1.01-2.09) and unfavourable outcome at discharge (RR 1.27, 1.02-1.60). In the validation cohort $(\mathrm{n}=379)$, pneumococcal aetiology (RR 1.69, 1.10-2.59) and abnormal cranial imaging (RR 1.68, 1.09-2.59) were confirmed as risk factors for seizures at any time. For seizures occurring after admission, only pneumococcal meningitis (RR 1.92, 1.12-3.29) remained significant. Seizures at any time were also associated with in-hospital mortality (RR 3.26, 1.83-5.80) and unfavourable outcome (RR 1.23, 1.00-1.52) in this cohort.

Conclusions Pneumococcal aetiology, immunocompromise and abnormal cranial imaging were risk factors for seizures in CABM. Seizures were strongly associated with mortality and unfavourable outcome.
Strengths and limitations of this study

- Using a two-cohort design, we first analysed the data in a retrospective population-based derivation cohort and next validated the results in a contemporary prospective nationwide population-based observational cohort.

- We defined risk factors and specified adjustments prior to data acquisition and analyses based on previous studies and biological reasoning.

- We included clinically meaningful risk factors amenable for future improvement such as antibiotic delay and treatment with adjunctive dexamethasone.

- Risk factors were also examined in stratified analyses of seizures occurring before or immediately at admission and seizures occurring during admission.

- Limitations inherent of retrospective designs also applied to our study including unavailability of electroencephalographs, random missing values and non-standardised treatment.

\section{INTRODUCTION}

Community-acquired bacterial meningitis $(\mathrm{CABM})$ is a devastating and often fatal condition. Seizures are frequent in CABM and have been associated with increased case fatality. ${ }^{1-5}$ The pathogenesis of seizures in CABM may involve inflammatory neuronal excitation, necrosis and increased intracranial pressure. A previous study compared patients with CABM complicated by seizures to those without seizures and found that they more often had cerebrospinal fluid (CSF) leucocytes $<1000 / \mathrm{mL}$, CSF protein $>3.0 \mathrm{~g} / \mathrm{L}$, increased erythrocyte sedimentation rate, a pneumococcal aetiology and focal cerebral abnormalities. ${ }^{1}$

Some authors advocate routine administration of anticonvulsive therapy in patients with suspected pneumococcal meningitis 
irrespective of whether or not seizures have occurred. ${ }^{2}$ This recommendation, however, has not been formally shown to improve the prognosis and is not adopted by most guidelines. ${ }^{6}{ }^{7}$ So far, few studies have specifically addressed risk factors and prognosis of seizures in CABM and previous results should be validated in other cohorts. A better understanding of clinical characteristics associated with increased risk of seizures could help define a population of adult patients eligible for a clinical trial of anticonvulsive therapy in CABM.

Therefore, we aimed to examine predefined risk factors for seizures in adult patients with CABM and the prognostic effect of seizures on outcome in a retrospective cohort. Next, we validated our results in a nationwide prospective bacterial meningitis cohort.

\section{MATERIAL AND METHODS}

\section{Setting and study population}

In Denmark, healthcare is financed by taxes and therefore free of charge at the point of delivery. All residents are assigned a unique 10-digit personal identification number at birth or immigration, which can be used to access information from all hospitalisations and other healthcare-related contacts.

\section{Derivation cohort}

We combined two retrospective cohorts of adult patients with CABM from North Region Denmark (1998-2014) and Nordsjællands and Hvidovre Hospitals (2003-2014) as described previously (figure 1). ${ }^{8}$ We used the electronic laboratory information systems to identify patients (ADBakt, Autonik Sweden at the Department of Clinical Microbiology at Aalborg University Hospital and LABKA II, CSC Scandihealth, Denmark, at the Departments of Clinical Biochemistry at Hvidovre and Nordsjællands Hospitals). We included all patients $>15$ years of age with a clinical presentation suggesting CABM and at least one of the following criteria ${ }^{8}$ :

1. Positive CSF culture.

2. Positive blood culture and CSF pleocytosis $>10$ leucocytes $/ \mathrm{mL}$.

3. Presence of bacteria on Gram stain of CSF.

4. Non-culture detection of bacteria in CSF by PCR-based technology or antigen analyses.

\section{Derivation cohort (Retrospective)}

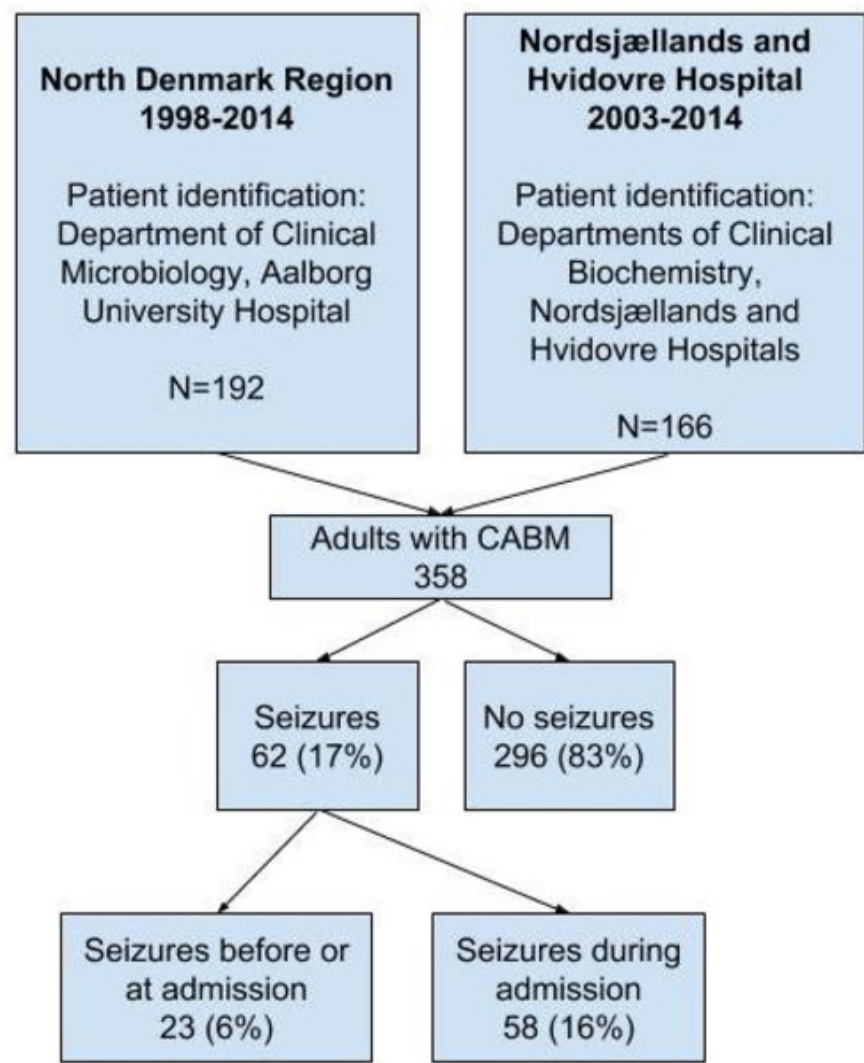

Validation cohort (Prospective)

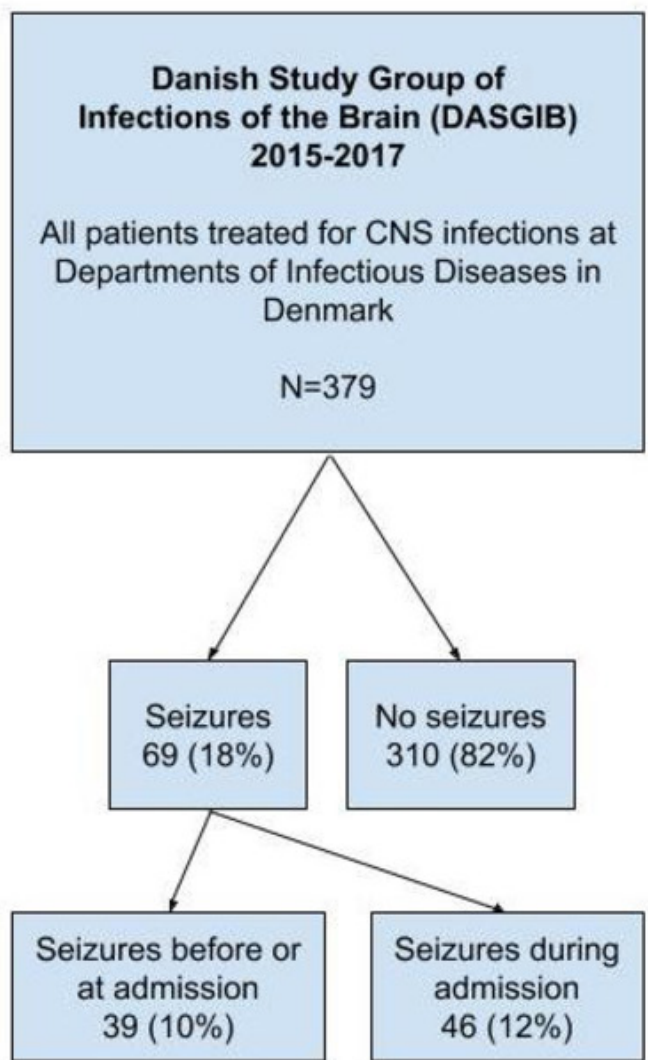

Figure 1 Flowchart of included patients with CABM in the derivation and validation cohorts. CABM, community-acquired bacterial meningitis; CNS, central nervous system. 
Exclusion criteria were previous neurosurgery, primary brain abscesses or hospital-acquired bacterial meningitis as defined by the Centers for Disease Control and Prevention. ${ }^{9}$

\section{Patient data}

We used the medical records to examine baseline characteristics including patient history, symptoms and signs at admission, time of diagnosis (at admission or later), adjuvant dexamethasone treatment and laboratory results (biochemistry, radiology, microbiology). For radiological results, we relied on the radiologist's descriptions. We defined immunocompromise as presence of any of the following comorbidities: alcohol abuse, asplenia, diabetes mellitus, renal impairment, liver cirrhosis, immunosuppressive therapy (eg, chemotherapy or high-dose prednisolone), solid or haematological cancer. Observation charts were obtained for patients who were treated in an intensive care unit (ICU). For patients with seizures, we included prior history of seizure disorder, use of anticonvulsive therapy as well as type, day(s) and cumulative incidence of seizures during admission for CABM. Electroencephalographs (EEGs) were not available in the medical records. We used the Glasgow Outcome Scale (GOS) score at discharge for assessment of outcome and categorised a GOS score of 5 as favourable, while GOS 1-4 were considered an unfavourable outcome.

\section{Validation cohort}

We used the Danish Study Group of Infections of the Brain (DASGIB) bacterial meningitis cohort to validate our results. ${ }^{10}$ Briefly, all adults with central nervous system infections treated by departments of infectious diseases in Denmark have been prospectively included in a database since 1 January 2015. The database contains information on baseline demographics, signs and symptoms at admission, time to antibiotic therapy for CABM, laboratory and imaging results, complications during admission and GOS scores at discharge. As in the derivation cohort, episodes of seizures analysed in this study were restricted to those occurring before or at some point during admission or stay at rehabilitation unit until discharge to home or nursing home. We validated our findings among patients with CABM registered in the DASGIB cohort from year 2015 through 2017. Prior to carrying out the current study, the DASGIB cohort was first registered on www.clinicaltrials.gov (NCT03418441) on February 1, 2018.

\section{Statistical analyses}

Baseline data are presented in contingency tables. Categorical variables are presented as proportions with percentages (\%) and examined by Fisher's exact test or $\mathrm{X}^{2}$, while continuous variables are presented as medians with IQR and compared by Mann-Whitney U-test.

Using modified Poisson regression analyses, we computed adjusted relative risks (RRs) with 95\% CIs for seizures at any time and for new onset seizures after admission only. ${ }^{11}$ Based on previous studies and biological plausibility, our prespecified variables consisted of: Age $>65$ years, immunocompromise, pneumococcal aetiology, time to antibiotic therapy for CABM within 2 hours of admission, adjunctive dexamethasone treatment and abnormal cranial imaging. ${ }^{1}$ Examining risk factors for new onset seizures after admission in the validation cohort, a limited number of events $(n=44)$ restricted the analysis to four prioritised variables: Age $>65$ years, immunocompromise, pneumococcal aetiology and abnormal cranial imaging.

Next, we used similar multivariable analyses to compute the prognostic value of seizures at any time during CABM on risk of death and unfavourable outcome at discharge. These analyses of outcome were adjusted for age $>65$ years, sex, diagnosis of meningitis immediately at admission, time to antibiotic therapy for CABM within 2 hours of admission, pneumococcal aetiology, adjuvant dexamethasone treatment and GCS $<15$ at admission. However, time of diagnosis was not available in the DASGIB database and was therefore omitted from the adjusted outcome analyses in this cohort. We also performed a sensitivity analysis of risk factors and outcome excluding the five patients with known seizure disorder.

We used STATA MP V.15.1 for all statistical analyses.

\section{Ethics}

The study was notified to the Danish Data Protection Agency in North Denmark Region (record no. 2008-580028) and Capital Region of Denmark (record no. 01706 hvh-2012-016, NOH-2015-012) and the Danish Health and Medicines Authority (record no. 3-3013-135/1 and 3-3013-2579/1). An approval from an ethics committee is not required for this type of study in Denmark.

\section{Patient and public involvement}

Neither patients nor public institutions were involved in this study. There are no plans of disseminating the results of this report to study participants besides publication in an open-access medical journal.

\section{RESULTS \\ Derivation cohort}

Seizures at any time during CABM occurred in 62 of 358 $(17 \%)$ patients in the derivation cohort of whom $23(6 \%)$ had seizures before or immediately at the time of admission (table 1).

Five $(9 \%)$ of these patients had a known seizure disorder before CABM. For 15 patients, the day and type of seizures in CABM were not specified in the medical records. The median time of first seizure episode in patients only having seizures during admission was day 1 (IQR 1-2). Types of seizures observed were generalised in 34 patients, focal in 10 of which 4 had secondary generalisation and non-convulsive status epilepticus in 3. The median number of days with seizures was 1 (IQR 1-2, range 1-23). Treatment for seizures was administered to 43 of $62(69 \%)$ patients of whom $30(48 \%)$ were given 
Table 1 Baseline characteristics of patients with community-acquired bacterial meningitis with and without seizures at any time during admission

\begin{tabular}{|c|c|c|c|}
\hline Baseline characteristics & $\begin{array}{l}\text { Seizures } \\
(n=62)\end{array}$ & No seizures $(n=296)$ & $P$ value \\
\hline Age (IQR), n=358 & $63(51-70)$ & $58.5(46-70)$ & 0.13 \\
\hline Female & $27 / 62(44)$ & $152 / 296(51)$ & 0.26 \\
\hline Immunocompromise, $n=358$ & 28/62 (45) & 95/296 (32) & 0.049 \\
\hline Diabetes mellitus & $7 / 62(11)$ & $17 / 296(6)$ & 0.11 \\
\hline Alcohol abuse & $11 / 62(18)$ & $39 / 296(13)$ & 0.35 \\
\hline Otitis/sinusitis/mastoiditis/pneumonia & $31 / 62(50)$ & $107 / 296(36)$ & 0.04 \\
\hline Duration of symptoms (days), $n=183$ & $2(2-3.5)$ & $2(2-5)$ & 0.42 \\
\hline \multicolumn{4}{|l|}{ Symptoms and signs on presentation } \\
\hline Headache & $36 / 49(73)$ & $175 / 234(75)$ & 0.85 \\
\hline Nausea/Vomiting & $21 / 45(47)$ & 93/201 (46) & 0.96 \\
\hline Neck stiffness & $38 / 58(66)$ & $180 / 273(66)$ & 0.95 \\
\hline Temperature $\left({ }^{\circ} \mathrm{C}\right), \mathrm{n}=327$ & $38.8(37.8-39.7)$ & $39.0(37.9-39.7)$ & 0.73 \\
\hline Petechiae & $10 / 48(21)$ & $49 / 216(23)$ & 0.78 \\
\hline Focal neurological deficits & $13 / 62(21)$ & $31 / 294(11)$ & 0.02 \\
\hline Glasgow Coma Scale $<15, n=358$ & $53(85)$ & $213(72)$ & 0.03 \\
\hline Diagnosed before or at admission, $\mathrm{n}=358$ & $37(60)$ & $208(70)$ & 0.10 \\
\hline Time to antibiotic therapy for meningitis since admission (hours), $n=333$ & $2.8(1.0-12)$ & $2.45(1-8)$ & 0.57 \\
\hline \multicolumn{4}{|l|}{ Laboratory results } \\
\hline C-reactive protein (mg/L), $\mathrm{n}=358$ & $204(108-327)$ & $215(115-303)$ & 0.74 \\
\hline B-leucocytes $\left(10^{9} / \mathrm{L}\right), \mathrm{n}=332$ & $17(10-25)$ & $17(12-23)$ & 0.67 \\
\hline B-thrombocytes $\left(10^{9} / L\right), n=307$ & $197(130-268)$ & $199(131-272)$ & 0.77 \\
\hline S-creatinine $(\mu \mathrm{mol} / \mathrm{L}), \mathrm{n}=318$ & $92(66-138)$ & $81(64-107)$ & 0.19 \\
\hline CSF leucocytes (median, IQR) $\left(10^{6} / \mathrm{L}\right), \mathrm{n}=346$ & $2806(287-6845)$ & $2110(483-5520)$ & 0.82 \\
\hline Protein in CSF (g/L), $\mathrm{n}=323$ & $4.3(2.1-9.1)$ & $3.6(1.8-6.5)$ & 0.09 \\
\hline CSF:blood glucose ratio (mmol/L), $n=323$ & $1.2(0.1-2.8)$ & $1.1(0.1-3.0)$ & 0.96 \\
\hline Positive blood culture, $\mathrm{n}=358$ & $41(66)$ & $167(56)$ & 0.29 \\
\hline Positive CSF culture, $\mathrm{n}=358$ & $51(82)$ & $247(83)$ & 0.34 \\
\hline Pneumococcal aetiology, $n=358$ & $43(69)$ & $151(51)$ & 0.008 \\
\hline Meningococcal aetiology, $n=358$ & $3(5)$ & $56(19)$ & 0.007 \\
\hline Adjunctive dexamethasone treatment, $n=358$ & $33(53)$ & $148(50)$ & 0.66 \\
\hline Intensive care unit treatment, $\mathrm{n}=358$ & $57(92)$ & $149(51)$ & $<0.001$ \\
\hline
\end{tabular}

Data presented as $\mathrm{n} / \mathrm{N}(\%)$ or medians (IQR).

Immunocompromise: alcohol abuse, asplenia, diabetes mellitus, renal impairment, liver cirrhosis, immunosuppressive therapy (eg, chemotherapy or high-dose prednisolone), solid or haematological cancer.

CSF, cerebrospinal fluid.

combination anticonvulsive therapy (9 patients if combinations with benzodiazepines were not included). Anticonvulsive treatments used included benzodiazepines in $30(48 \%)$ patients, sedation in the ICU in $17(27 \%)$, valproate in $11(18 \%)$, phenytoin in $5(8 \%)$, lamotrigine in $2(3 \%)$, levetiracetam in $2(3 \%)$, oxcarbazepine and carbamazepine in 1 each $(2 \%)$.

Comparing patients with seizures at any time during CABM to patients without seizures, we observed no substantial differences in most presenting symptoms or baseline blood and CSF biochemistry (table 1). However, focal neurological deficits (13 of $62(21 \%)$ vs 31 of 296 $(11 \%))$ and altered mental status (53 of $62(85 \%)$ vs 213 of $296(72 \%))$ were more common in these patients than in patients without seizures. Seizures occurred in 43 of 194 (22\%) patients with pneumococcal meningitis compared with 19 of 145 (13\%) patients with other aetiologies (table 2).

Conversely, seizures were only observed among 3 of 59 (5\%) patients with meningococcal meningitis. Abnormalities in cranial imaging were found in 28 of 57 (48\%) patients with seizures compared with 62 of 209 (30\%) 
Table 2 Causative pathogens of community-acquired bacterial meningitis in patients with and without seizures at any time during admission

\begin{tabular}{llc}
\hline Bacterial aetiology & $\begin{array}{l}\text { Seizures } \\
(\mathbf{n = 6 2 )}\end{array}$ & $\begin{array}{l}\text { No seizures } \\
(\mathbf{n = 2 9 6 )}\end{array}$ \\
\hline Streptococcus pneumoniae & $43(69)$ & $151(51)^{\star}$ \\
\hline Neisseria meningitidis & $3(5)$ & $56(19)^{\star}$ \\
\hline Listeria monocytogenes & $4(6)$ & $22(7)$ \\
\hline Beta-haemolytic streptococci & $4(6)$ & $10(3)$ \\
(A, B, C, G) & & \\
\hline Non-haemolytic streptococci & $2(3)$ & $6(2)$ \\
\hline Staphylococcus aureus & $1(2)$ & $20(7)$ \\
\hline Escherichia coli & - & $8(3)$ \\
\hline Haemophilus influenzae & $3(5)$ & $13(4)$ \\
\hline Capnocytophaga canimorsus & - & $5(2)$ \\
\hline Klebsiella pneumonia & - & $1(1)$ \\
\hline Enterococcus spp. & $1(2)$ & $3(1)$ \\
\hline Pasteurella multocida & $1(2)$ & - \\
\hline Chlamydia pneumoniae & - & $1(1)$ \\
\hline
\end{tabular}

Data are presented as $\mathrm{n}(\%)$.

${ }^{*} P$ value $<0.05$

patients without seizures with infarction as the most common finding (table 3 ).

Twenty-two of $62(35 \%)$ patients with seizures died during admission for CABM versus 62 of 296 (21\%) in patients without seizures, while unfavourable outcome at discharge occurred in 39 of $62(63 \%)$ versus 127 of $296(43 \%)$ patients (table 4$)$. There was no difference in fatal outcome among patients with seizures who received anti-epileptic treatment and those who did not (17 of 43 $(40 \%)$ versus 5 of 19 (26\%) patients, $\mathrm{p}=0.32)$.

Examining risk factors for seizures at any time during admission by univariable and multivariable analyses, we
Table 4 Outcomes of patients with community-acquired bacterial meningitis with or without seizures at any time during admission

\begin{tabular}{lll}
\hline Outcome & $\begin{array}{l}\text { Seizures } \\
(\mathbf{n}=62)\end{array}$ & $\begin{array}{l}\text { No seizures } \\
(\mathbf{n}=\mathbf{2 9 6})\end{array}$ \\
\hline $\begin{array}{l}\text { Glasgow Outcome Scale score } \\
1\end{array}$ & $22(35)$ & $62(21)^{\star}$ \\
\hline 2 & - & $2(1)$ \\
\hline 3 & $7(11)$ & $28(9)$ \\
4 & $10(16)$ & $35(12)$ \\
\hline 5 & $23(37)$ & $169(57)^{\star}$ \\
$\begin{array}{l}\text { Seizures after discharge/subsequent } \\
\text { epilepsy }\end{array}$ & $7 / 62(13)$ & $\mathrm{Na}$ \\
\hline Duration of admission (days), $\mathrm{n}=358$ & $21(12-36)$ & $14(10-23)^{\star}$ \\
\hline Time to death (days), $\mathrm{n}=358$ & $7(3-27)$ & $8(3-16)$ \\
\hline
\end{tabular}

Data are presented as $\mathrm{n}(\%)$ or medians (IQR).

${ }^{*} P$ value $<0.05$

$\mathrm{Na}$, not available.

observed an increased risk in patients with pneumococcal aetiology (adjusted RR 1.69, 95\% CI 1.02 to 2.83) and abnormal cranial imaging (adjusted RR 2.27, 95\% CI 1.46 to 3.53) compared with patients without these characteristics (tables 5 and 6 ).

Restricting the multivariable analysis to patients with seizures during admission, only abnormal cranial imaging (adj. RR 1.90, 95\% CI 1.21 to 2.98) and immunocompromise (adj. RR 1.62, 95\% CI 1.01 to 2.57 ) were significantly associated with increased risk, while pneumococcal aetiology also suggested an association (adj. RR 1.65, 95\% CI 0.99 to 2.76). Significant associations between seizures and abnormal cranial imaging were confirmed in stratified analyses of pneumococcal aetiology (data not shown).

Seizures at any time during CABM increased the risk of case fatality by $45 \%$ (adj. RR $1.45,95 \%$ CI 1.01 to 2.09 ) and the risk of unfavourable outcome at discharge by

Table 3 Cranial imaging in patients with community-acquired bacterial meningitis with or without seizures at any time during admission

\begin{tabular}{llll}
\hline Baseline characteristics & $\begin{array}{l}\text { Seizures } \\
(\mathbf{n}=\mathbf{5 7})\end{array}$ & No seizures $\mathbf{( n = 2 0 9 )}$ & P value \\
\hline Abnormal cranial imaging & $28(49)$ & $62(30)$ & 0.006 \\
Infarction & $15(26)$ & $23(11)$ & 0.003 \\
Generalised brain swelling & $7(12)$ & $15(7)$ & 0.22 \\
Haemorrhage & $4(7)$ & $14(7)$ & 0.93 \\
\hline Hydrocephalus & 0 & $10(5)$ & 0.09 \\
Ventriculitis & $1(2)$ & $5(2)$ & 0.77 \\
Cerebritis & $1(2)$ & $4(2)$ & 0.94 \\
Malignant tumour & $1(2)$ & $2(1)$ & 0.61 \\
Secondary brain abscess/empyema & $2(4)$ & $1(1)$ & 0.06 \\
Sinus thrombosis & $1(2)$ & $1(1)$ & 0.32 \\
\hline
\end{tabular}

Data are presented as $\mathrm{n}(\%)$. 
Table 5 Univariate analysis for risk factors of seizures in CABM using patients with no seizures as reference

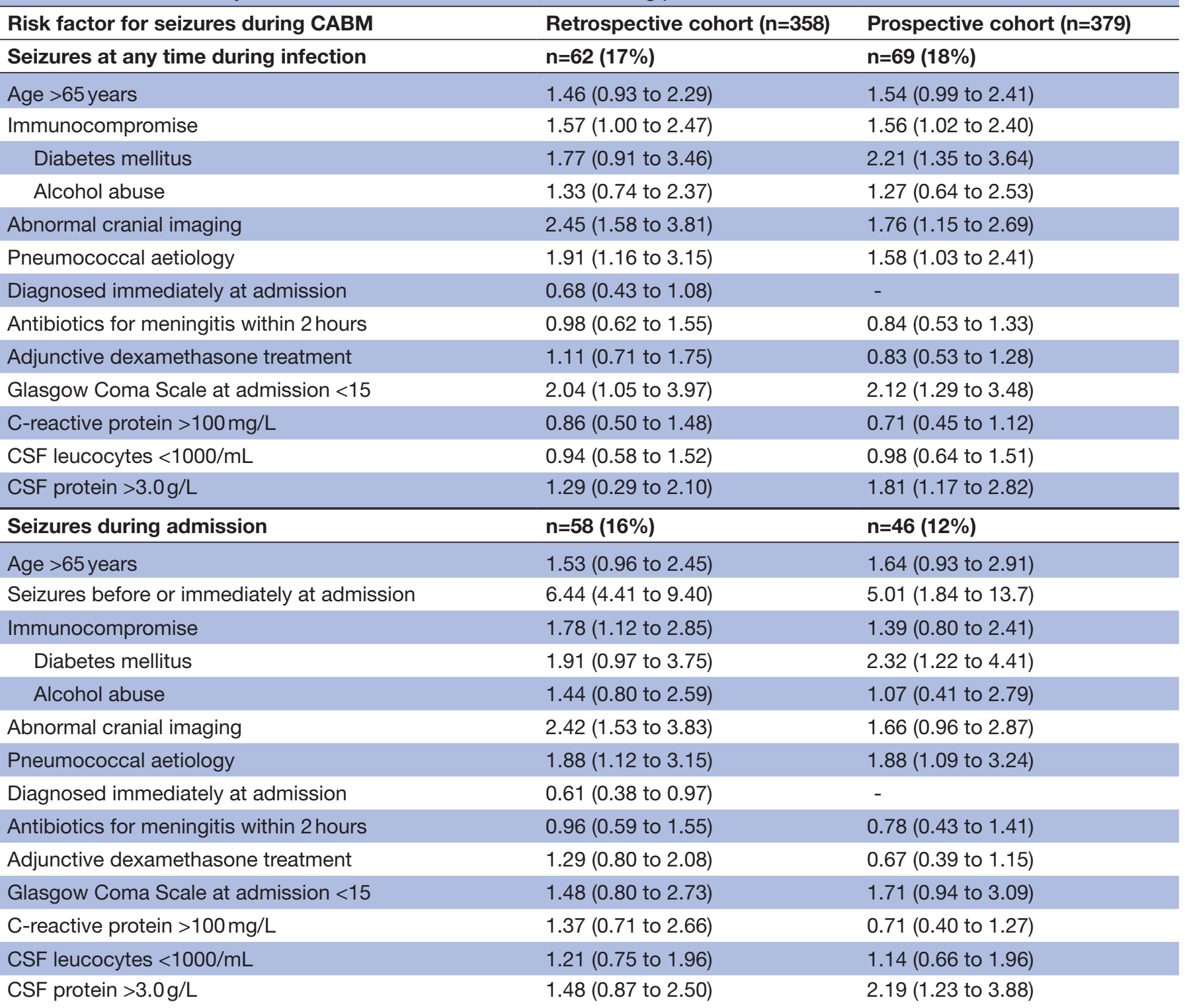

Data are presented as relative risks with $95 \% \mathrm{Cl}$.

CABM, community-acquired bacterial meningitis; CSF, cerebrospinal fluid.

$27 \%$ (adj. RR $1.27,95 \%$ CI 1.02 to 1.60 ). Stratified analyses of pneumococcal meningitis patients with seizures at any time during admission compared with those without seizures showed an increased case fatality (14 of 43 (33\%) vs 31 of $151(21 \%), \mathrm{p}=0.10)$ and risk of unfavourable outcome at discharge (28 of $43(65 \%)$ vs 70 of $151(46 \%)$, $\mathrm{p}=0.03)$.

\section{Validation cohort}

Next, we validated our results among 379 adult patients (47\% female) with CABM in the DASGIB cohort. The median age was 66 years (IQR 54-74) and seizures at any time during admission occurred in 69 of $379(18 \%)$ patients. The aetiology was predominated by Streptococcus pneumoniae (41\%) followed by Staphylococcus aureus (10\%) and beta-haemolytic streptococci (5\%). A fatal outcome was observed in 50 of $379(13 \%)$ patients, while an unfavourable outcome at discharge was found in 199 of 379 patients $(53 \%)$ in this cohort.

We confirmed that pneumococcal aetiology (adj. RR $1.69,95 \%$ CI 1.10 to 2.59 ) and abnormal cranial imaging (adj. RR 1.68, 95\% CI 1.09 to 2.59) were associated with increased risks of seizures at any time during admission (table 6). When we restricted the analysis to seizures after admission, only pneumococcal aetiology remained associated with increased risks (adj. RR 1.92, 95\% CI 1.12 to 3.29). Seizures at any time during CABM increased the risk for case fatality by $226 \%$ (adj. RR $3.26,95 \%$ CI 1.83 to 5.80 ) and for unfavourable outcome at discharge by $23 \%$ (adj. RR 1.23, 95\% CI 1.00 to 1.52 ). 
Table 6 Multivariate analyses for risk factors of seizures in CABM using patients with no seizures as reference

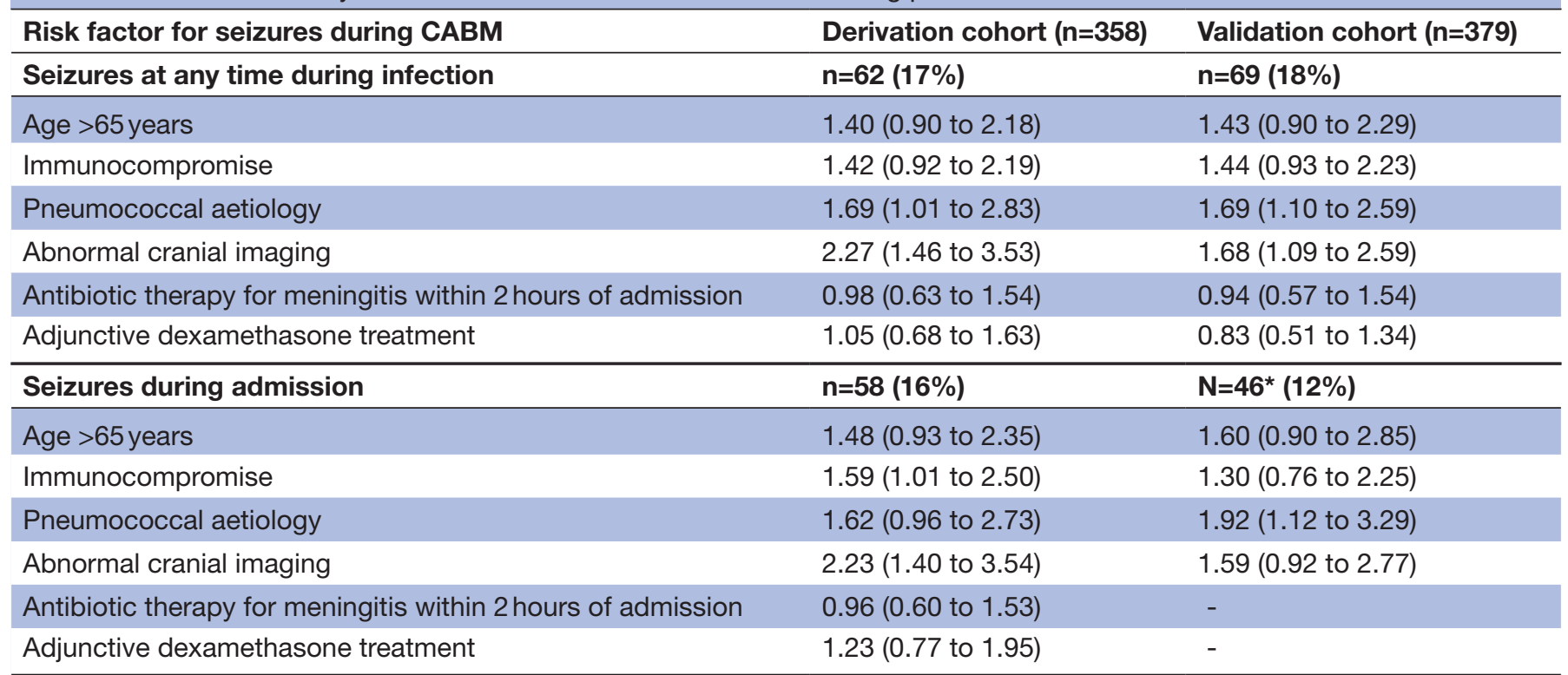

Data are presented as relative risks with $95 \% \mathrm{Cl}$.

${ }^{\star}$ The limited number of events in this group restricted the number of variables included in the adjusted model as described under 'Statistical analyses'.

CABM, community-acquired bacterial meningitis.

Supplementary analyses in both the derivation and validation cohorts revealed no substantial effects of time to antibiotic therapy for CABM within 2 hours of admission or adjunctive dexamethasone on risks of seizures in CABM (tables 5 and 6).

Sensitivity analyses excluding patients with known seizure disorder $(n=5)$ did not change the overall results of risk factors or prognosis in either cohort.

\section{DISCUSSION}

We examined the association between several predefined variables and risk of seizures in adult CABM in two separate observational cohorts and identified pneumococcal meningitis, immunocompromise and abnormal cranial imaging (cerebral infarctions) as important risk factors. In addition, seizures were strongly associated with mortality and unfavourable outcome at discharge.

\section{Comparisons with other studies}

Few previous studies have specifically addressed risk factors for seizures in bacterial meningitis. ${ }^{1-5}$ A prospective Dutch observational study examined 696 episodes of CABM from 1998 through 2002 and found that 121 $(17 \%)$ of patients had seizures at some point during infection, ${ }^{1}$ which is identical to the findings in our study. They also observed several differences between patients with and without seizures by univariate analyses, for example, in CSF biochemistry. However, in multivariable analyses, only immunocompromise and pneumococcal meningitis remained strongly associated with seizures at any time with the addition of focal cerebral abnormalities (defined as aphasia, monoparesis, hemiparesis or quadriparesis) for seizures after 48 hours of admission. The focal cerebral abnormalities may be correlates of brain infarctions as suggested by the high prevalence among patients with seizures compared with patients without seizures $(20 \%$ vs $11 \%)$. Therefore, we consider the overall results comparable to ours with small differences in the methodology.

In our study, brain infarctions were found in $26 \%$ of CABM patients with seizures and $11 \%$ of those without seizures. In comparison, the incidence of seizures within 2 weeks among patients admitted with stroke typically ranges from $2 \%$ to $6.5 \%{ }^{12}$ Of relevance for the pathogenesis of brain infarction in CABM, a previous autopsy study of patients with pneumococcal meningitis documented extensive infarction and thrombosis in such patients and the authors hypothesised that infectious cerebral vasculitis or inflammation mediated by deposition of immune-complexes may be involved. ${ }^{13}$ This could potentially explain some of the observed associations between seizures, cerebral infarction and pneumococcal meningitis observed in our study. Further support may be derived by cerebral oedema or other intracranial complications being infrequent in patients with and without seizures in our study. Of note, studies remain inconclusive regarding the role of adjunctive dexamethasone in the rare occurrence of delayed-onset stroke after bacterial meningitis. ${ }^{14} 15$

Two other studies reported seizures more often among elderly ( $>65$ and 60 years of age, respectively) than in younger CABM patients, but multivariable analyses of risk factors for seizures were not reported. ${ }^{2}{ }^{16}$ Although the point estimates in our univariate and multivariate analyses consistently suggest age $>65$ years as a potential risk factor for seizures in CABM, the confidence intervals 
indicate too much statistical uncertainty for this to be a confirmed risk factor for now.

Similar to our study, other reports have examined seizures as a prognostic factor in bacterial meningitis and found increased risks of case fatality and unfavourable outcome. ${ }^{2}$ 16-18 $^{16}$ A prognostic model based on 176 patients with CABM from 1970 through 1995 showed an increased risk of in-hospital mortality or neurological deficits at discharge in patients with seizures at admission (OR 4.42, 95\% CI 1.56 to 12.48). ${ }^{17}$ The model was adjusted for immune-competence, comorbidity and CSF leucocyte count and subsequently validated in a second cohort. Another study also observed an increased risk of in-hospital mortality in univariate analysis of bacterial meningitis patients with seizures within 24 hours of admission (RR 4.0, 95\% CI 2.8 to 5.8). ${ }^{4}$ Likewise, a third study found seizures occurring after treatment initiation for CABM in elderly patients to be associated with in-hospital mortality (OR 6.8, 95\% CI 1.7 to 27 ). ${ }^{2}$ The authors also state a protective effect of several variables including anti-seizure prophylaxis, but neither these results nor the statistical models were presented.

Finally, a large study of 352 patients with pneumococcal meningitis found an equal distribution of seizures at presentation among those with favourable outcome $(8 \%)$ and unfavourable outcome $(6 \%) .{ }^{19}$ This suggests that some of the observed prognostic effect of seizures on mortality and unfavourable outcome in our study could be related to seizures after admission or pneumococcal aetiology, even though we adjusted for the latter in our models.

\section{Limitations}

Several conditions could result in an underestimation of the cumulative incidence of seizures in CABM. These include patients admitted comatose without a detailed patient history, requests for cranial imaging before lumbar puncture with the risk of death before the diagnosis is confirmed (by lumbar puncture) or because a contraindication to lumbar puncture is present, for example, intracranial mass lesion. On the other hand, antibiotics used for CABM have been associated with a low absolute risk of seizures, which may lead to an increase in the incidence of seizures in these patients. However, we consider this negligible compared with the effect of severe inflammation of the meninges, high fever, brain infarctions and sepsis.

The categorisation of seizures into those occurring before or immediately at admission and those after admission is arbitrary, and may be influenced by, for example, time to diagnosis and disease severity. However, the distinction has clinical relevance, as seizures occurring after admission could be a target for prevention. Compared with patients without seizures, patients with seizures more often had cranial imaging performed in our study and were therefore more likely to be diagnosed with, for example, brain infarctions. Still, other abnormalities on cranial imaging did not differ suggesting a true difference in occurrence of clinically relevant infarctions between the two patient groups. Moreover, the occurrence of brain infarctions was similar in frequency to another study in which the cranial imaging was performed equally often among patients with and without seizures during CABM. ${ }^{1}$ A point of consideration is that MRI is more sensitive than CT in detecting brain infarctions but it was seldom used in our patients. This may have led to an underestimation in the incidence of intracranial abnormalities.

Other limitations inherent of observational studies also apply to our results. Some details concerning, for example, number and type of seizures as well as type of cranial imaging or distribution of infarctions were not always well described in the medical records. Further, reports of EEG were unavailable, which may underestimate the incidence of subclinical or non-convulsive status among patients with impaired consciousness Nonetheless, both frequency and seizure characteristics were comparable to a previous study with such information available. ${ }^{1}$ Missing values were accounted for by providing exact numbers of observations in the tables. Unfortunately, we only had sporadic information on intracranial pressures and were unable to perform meaningful analysis of associations with occurrence of seizures. Finally, the treatment of patients was performed by the responsible physician and may as such be subject to variation.

\section{Implications}

We agree with Zoons et al that a low threshold for anticonvulsant therapy in patients with seizures in CABM is prudent. ${ }^{1}$ Although the observed mortality with seizures in patients with CABM is high, causality cannot be inferred by observational studies and the associations could be due to, for example, pneumococcal aetiology, infarctions or disease severity. Therefore, routine anticonvulsive treatment to patients with CABM is not advised for now. Non-convulsive status was rare in our and other studies, but should be considered in patients with unexplained fluctuating consciousness or those who do not regain consciousness. ${ }^{1}$ Prolonged or intensified anti-inflammatory treatment could be considered in future experimental studies if infectious cerebral vasculitis is confirmed as an important contributor to infarctions, seizures and fatal outcome in a substantial part of patients with pneumococcal meningitis. Our observations were confirmed in a separate prospective cohort, and the characteristics associated with risk of seizures and adverse outcome could, together with findings in other studies, help define a patient population eligible for clinical trials of prophylactic anti-epileptic drugs in adults with CABM.

\section{Author affiliations}

${ }^{1}$ Department of Infectious Diseases, Aalborg University Hospital, Aalborg, Denmark

${ }^{2}$ Department of Infectious Diseases, Nordsjællands Hospital, Hillerod, Denmark ${ }^{3}$ Department of Infectious Diseases, Odense University Hospital, Odense, Denmark ${ }^{4}$ Department of Infectious Diseases, Aarhus University Hospital Skejby, Aarhus, Denmark 
${ }^{5}$ Department of Infectious Diseases, Sjællands Universitetshospital Roskilde, Roskilde, Denmark

${ }^{6}$ Department of Infectious Diseases, Rigshospitalet, Kobenhavn, Denmark ${ }^{7}$ Department of Infectious Diseases, Hvidovre Hospital, Hvidovre, Denmark ${ }^{8}$ Department of Clinical Microbiology, Hvidovre Hospital, Hvidovre, Denmark

Correction notice This article has been corrected since it was published. Author name has been updated.

Acknowledgements Thanks to all departments of infectious diseases in Denmark who participate in DASGIB.

Collaborators Merete Storgaard (member of the DASGIB study group; e-mail merestor@rm.dk)

Contributors JB conceived the study. FTBDL, CTB, LL, VK, LW, JHL, BRH and JB participated in collection of data. FTBDL and JB analysed the data. FTBDL, CTB, $\mathrm{LL}, \mathrm{C} \emptyset \mathrm{A}, \mathrm{HN}$ and JB interpreted the data. FTBDL wrote the first draft. CTB, LL, VK, $\mathrm{LW}, \mathrm{JHL}, \mathrm{BRH}, \mathrm{C} \emptyset \mathrm{A}, \mathrm{HN}$ and JB critically reviewed the manuscript and all authors (FTBDL, CTB, LL, VK, LW, JHL, BRH, CØA, HN and JB) approved submission.

Funding The authors have not declared a specific grant for this research from any funding agency in the public, commercial or not-for-profit sectors.

Competing interests None declared.

Patient consent for publication Not required.

Provenance and peer review Not commissioned; externally peer reviewed.

Data sharing statement Permissions from the Danish Data Protection Agency and the Danish Board of Health are required before anonymised data can be shared by request from a qualified researcher.

Open access This is an open access article distributed in accordance with the Creative Commons Attribution Non Commercial (CC BY-NC 4.0) license, which permits others to distribute, remix, adapt, build upon this work non-commercially, and license their derivative works on different terms, provided the original work is properly cited, appropriate credit is given, any changes made indicated, and the use is non-commercial. See: http://creativecommons.org/licenses/by-nc/4.0/.

\section{REFERENCES}

1. Zoons E, Weisfelt M, de Gans J, et al. Seizures in adults with bacterial meningitis. Neurology 2008;70:2109-15.

2. Cabellos C, Verdaguer R, Olmo M, et al. Community-acquired bacterial meningitis in elderly patients. Medicine 2009;88:115-9.
3. van de Beek D, de Gans J, Spanjaard L, et al. Clinical features and prognostic factors in adults with bacterial meningitis. N Engl J Med 2004;351:1849-59.

4. Durand ML, Calderwood SB, Weber DJ, et al. Acute bacterial meningitis in adults. A review of 493 episodes. N Engl J Med 1993;328:21-8.

5. Kastenbauer S, Pfister HW. Pneumococcal meningitis in adults: spectrum of complications and prognostic factors in a series of 87 cases. Brain 2003;126:1015-25.

6. van de Beek D, Cabellos C, Dzupova O, et al. ESCMID guideline: diagnosis and treatment of acute bacterial meningitis. Clin Microbiol Infect 2016;22 Suppl 3:1-26.

7. McGill F, Heyderman RS, Michael BD, et al. The UK joint specialist societies guideline on the diagnosis and management of acute meningitis and meningococcal sepsis in immunocompetent adults. $J$ Infect 2016;72:405-38.

8. Bodilsen J, Brandt CT, Sharew A, et al. Early versus late diagnosis in community-acquired bacterial meningitis: a retrospective cohort study. Clin Microbiol Infect 2018;24:166-70.

9. Garner JS, Jarvis WR, Emori TG, et al. CDC definitions for nosocomial infections, 1988. Am J Infect Control 1988;16:128-40.

10. Bodilsen J, Storgaard M, Larsen L, et al. Infectious meningitis and encephalitis in adults in Denmark: a prospective nationwide observational cohort study (DASGIB). Clin Microbiol Infect 2018;24:1102.e1-5.

11. Zou G. A modified poisson regression approach to prospective studies with binary data. Am J Epidemiol 2004;159:702-6.

12. Camilo O, Goldstein LB. Seizures and epilepsy after ischemic stroke. Stroke 2004;35:1769-75.

13. Engelen-Lee J-Y, Brouwer MC, Aronica E, et al. Delayed cerebral thrombosis complicating pneumococcal meningitis: an autopsy study. Ann Intensive Care 2018;8:1-11.

14. Lucas MJ, Brouwer MC, van de Beek D. Delayed cerebral thrombosis in bacterial meningitis: a prospective cohort study. Intensive Care Med 2013;39:866-71.

15. Schut ES, Brouwer MC, de Gans J, et al. Delayed cerebral thrombosis after initial good recovery from pneumococcal meningitis. Neurology 2009;73:1988-95.

16. Weisfelt M, van de Beek D, Spanjaard L, et al. Communityacquired bacterial meningitis in older people. J Am Geriatr Soc 2006;54:1500-7.

17. Aronin SI, Peduzzi P, Quagliarello VJ. Community-acquired bacterial meningitis: risk stratification for adverse clinical outcome and effect of antibiotic timing. Ann Intern Med 1998;129:862-9.

18. Lai WA, Chen SF, Tsai NW, et al. Clinical characteristics and prognosis of acute bacterial meningitis in elderly patients over 65 : a hospital-based study. BMC Geriatr 2011;11:91.

19. Weisfelt M, van de Beek D, Spanjaard L, et al. Clinical features, complications, and outcome in adults with pneumococcal meningitis: a prospective case series. Lancet Neurol 2006;5:123-9. 